\title{
ADDENDUM
}

\section{Fast cavity auto-tuning system for hydrogen masers}

\author{
C. Audoin \\ Laboratoire de l'Horloge Atomique, Equipe de Recherche du C.N.R.S., associée à l'Université Paris-Sud, \\ Bât. 221, Université Pariş-Sud, 91405 Orsay, France
}

In the derivation of the properties of a fast cavity autotuning system for active hydrogen masers [1], it has been implicitely assumed that the carrier of the signal injected into the microwave cavity is suppressed. Otherwise, the maser oscillation could be synchronized to this spectral component of the signal used to tune the microwave cavity.

In this Note, the condition which must be fulfilled to eliminate the considered carrier is specified.

Let $f(t)$ defined as :

$$
f(t)=\mathrm{e}^{i \varphi(t)}
$$

where the phase $\varphi(t)$ is periodic and is given by :

$\varphi(t)=\left(\omega_{\mathrm{r}}+\omega_{\mathrm{m}}\right) t-\frac{1}{4} \omega_{\mathrm{m}} T$ for $0<t<T / 2$ $\varphi(t)=\left(\omega_{\mathrm{r}}-\omega_{\mathrm{m}}\right) t+\frac{3}{4} \omega_{\mathrm{m}} T$ for $T / 2<t<T$.

The quantity $T$ is the period of the modulation, whose angular frequency is $\omega_{\mathrm{f}}=2 \pi / T$.

It can be seen easily that the frequency $\omega(t)$ of signal $f(t)$ is square-wave modulated, such as :

$$
\begin{aligned}
& \omega(t)=\omega_{\mathrm{r}}+\omega_{\mathrm{m}} \text { for } 0<t<T / 2 \\
& \omega(t)=\omega_{\mathrm{r}}-\omega_{\mathrm{m}} \text { for } T / 2<t<T
\end{aligned}
$$

The function $f(t)$ is the complex representation of the time dependent part of the signal considered in reference [1]. It can be expanded in a Fourier series as follows :

$$
f(t)=\mathrm{e}^{i \omega_{\mathrm{r}} t} \sum_{k=-\infty}^{\infty} \lambda_{k} \mathrm{e}^{i k \omega_{\mathrm{f}} t}
$$

where $k$ is an integer. For $k \neq \pm m$, coefficient $\lambda_{k}$ is given by :

$$
\begin{aligned}
\lambda_{k} & =\frac{2 m}{\pi\left(m^{2}-k^{2}\right)} \times \\
& \times\left[\cos ^{2} k \frac{\pi}{2} \cdot \sin m \frac{\pi}{2}+i \sin ^{2} k \frac{\pi}{2} \cdot \cos m \frac{\pi}{2}\right]
\end{aligned}
$$

and for $k= \pm m$, we have :

$$
\lambda_{k}=\frac{1}{2}\left[\cos \frac{\pi}{2} m-i \sin \frac{\pi}{2} m\right] .
$$

The parameter $m$ is the modulation index defined as follows :

$$
m=\frac{\omega_{\mathrm{m}}}{\omega_{\mathrm{f}}}
$$

Equation (5) shows that the amplitude of the carrier is proportional to $\sin m \frac{\pi}{2}$. It is naught for $m=2 p$, where $p$ is an integer. The carrier is then suppressed if the modulation depth $\omega_{m}$ is an even multiple of the modulation frequency $\omega_{\mathrm{f}}$. This condition can be exactly satisfied using the techniques of frequency synthesis, and the carrier can be completely eliminated.

Such a result would be much more difficult to achieve with sine-wave modulation where the carrier is suppressed for an irrational value of the modulation index.

\section{Reference}

[1] Audorn, C., Revue Phys. Appl. 16 (1981) 125. 